\title{
Transmitral inflow wave and progression from paroxysmal to permanent atrial fibrillation in Asian people
}

\author{
Takashi Nakagawa, ${ }^{1,2}$ Hisao Hara, ${ }^{1}$ Masaya Yamamoto, ${ }^{1}$ Yumi Matsushita, ${ }^{3}$ \\ Yukio Hiroi ${ }^{1}$
}

'Department of Cardiology, National Center for Global Health and Medicine, Tokyo, Japan

${ }^{2}$ Department of Cardiovascular Medicine, Tokyo Medical and Dental University, Tokyo, Japan ${ }^{3}$ Department of Clinical Research, National Center for Global Health and Medicine, Tokyo, Japan

\section{Correspondence to} Dr Takashi Nakagawa, Cardiology, National Center for Global Health and Medicine, Tokyo 162-8655, Japan; tnakagawa@hosp.ncgm.go.jp

Received 26 December 2018 Revised 30 March 2019 Accepted 22 May 2019

\section{Check for updates}

(c) Author(s) (or their employer(s)) 2019. No commercial re-use. See rights and permissions. Published by BMJ.

To cite: Nakagawa T, Hara $\mathrm{H}_{\text {, }}$ Yamamoto $\mathrm{M}$, et al. Heart Asia 2019:11:e011166. doi:10.1136/

heartasia-2018-011166

\section{ABSTRACT}

Objective Paroxysmal atrial fibrillation could progress to permanent atrial fibrillation. Whether the transmitral inflow waves could be used to predict progression from paroxysmal atrial fibrillation to permanent atrial fibrillation is unknown. Therefore, we investigated the association between the transmitral inflow waves and progression of paroxysmal atrial fibrillation.

Method We performed a retrospective study by analysing clinical and echocardiographic data from 88 patients with paroxysmal atrial fibrillation. We excluded patients who had structural heart disease, significant valvular disease, cardiomyopathy, cardiac device implantation or a left ventricular ejection fraction $<50 \%$. Result The patients with progression to permanent atrial fibrillation were more likely to be male and had lower peak $A$ velocity than those without progression. After adjusting for covariates, lower peak A velocity remained the independent predictor of progression to permanent atrial fibrillation $(p=0.025)$.

Conclusion The A velocity could be useful for predicting progression to permanent atrial fibrillation in Asian people.

\section{INTRODUCTION}

Atrial fibrillation (AF) is the most common cardiac arrhythmia encountered in clinical practice. The prevalence of AF is increasing with the ageing of populations in developed countries since it is more common among the elderly. ${ }^{1-3}$ Although AF is sometimes asymptomatic, this arrhythmia is associated with stroke and heart failure and, therefore, with increased morbidity and mortality. Thus, appropriate treatment of AF is required, such as anticoagulant therapy and rhythm or rate control therapy. $\mathrm{AF}$ is classified into three categories, which are paroxysmal, persistent and permanent. ${ }^{4}$ It has been reported that paroxysmal atrial fibrillation (PAF) undergoes progression to chronic AF unresponsive to therapy: permanent $\mathrm{AF}$ (PeAF) at an annual rate of $5.0 \%-8.6 \%$ and that $25 \%$ of patients with PAF develop PeAF after 5 years. ${ }^{5}$ Both electrophysiological and structural remodelling may contribute to the onset and maintenance of AF. ${ }^{6}$ The factors that cause progression from PAF to PeAF are not well established. Doppler echocardiography can measure blood inflow velocity into the left ventricle across the mitral valve, including the peak early inflow velocity $(\mathrm{E}: \mathrm{cm} / \mathrm{s})$ and peak late or atrial inflow velocity $(\mathrm{A}: \mathrm{cm} / \mathrm{s})$, with the latter parameter being dependent on atrial contraction. These transmitral

\section{Key messages}

What is already known about this subject?

- The prevalence of atrial fibrillation is increasing with the ageing of populations in developed countries. Paroxysmal atrial fibrillation undergoes progression to permanent atrial fibrillation. However, the factors that cause the progression to permanent atrial fibrillation are not well established.

What does this study add?

- This study demonstrates that the simple parameter of echocardiography might be useful for prediction of progression to permanent atrial fibrillation. Low peak A velocity could predict the progression.

How might this impact on clinical practice?

- To identify the predictive factor for progression from paroxysmal atrial fibrillation to permanent atrial fibrillation may be helpful to delay the progression. We could maintain sinus rhythm safely by rhythm control therapy in patients with low risk of the progression, and we could give priority to rate control therapy for patients with an increased risk of the progression.

inflow waves have been used as an index of diastolic dysfunction. When left ventricular relaxation becomes abnormal, the left atria-left ventricle pressure gradient in the early diastolic phase decreases and $\mathrm{E}$ waves decrease. The blood, which remained in the left atrium, flows into the left ventricle by the effective left atrial contraction, and $\mathrm{A}$ waves increase. The transmitral inflow waves have been reported to be a predictor of new-onset $\mathrm{AF}^{78}$ but it is unclear whether the transmitral inflow waves could predict progression from PAF to PeAF. The aim of this study is to investigate whether the transmitral inflow wave indexes could be useful for predicting progression from PAF to PeAF.

\section{METHODS}

\section{Study setting}

This is a retrospective study that enrolled outpatients diagnosed with PAF between September 2010 and June 2013 at the department of cardiology in the Center Hospital of the National Center for Global Health and Medicine (Tokyo, Japan). Patients with PAF were eligible for inclusion if 


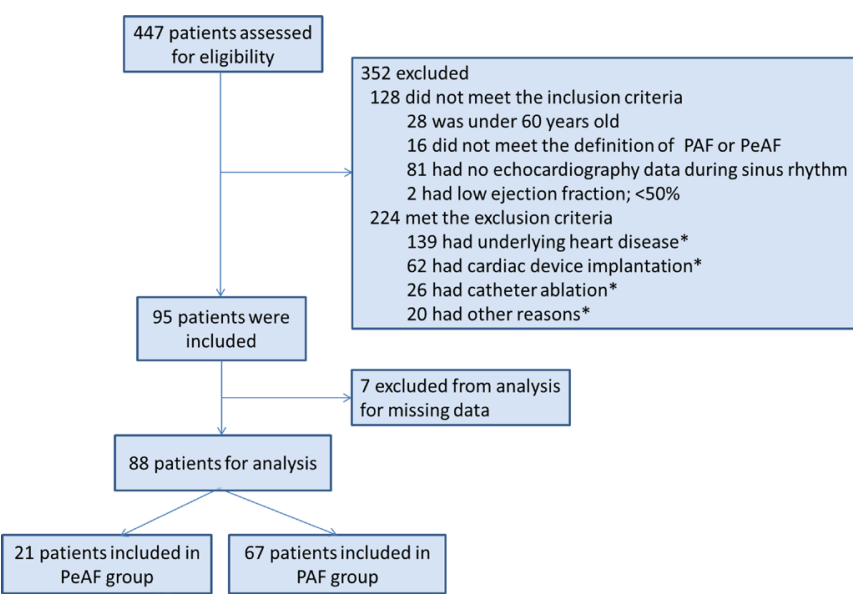

Figure 1 Flow of patients. *The criteria sometimes overlap with each other. PAF, paroxysmal atrial fibrillation; PeAF, permanent atrial fibrillation.

they were aged 60 years or older, but were excluded if they had structural heart disease, significant valvular disease (defined as moderate/severe stenosis or regurgitation affecting any heart valve on Doppler echocardiography), cardiomyopathy, cardiac device implantation, B-type natriuretic peptide $>200 \mathrm{pg} / \mathrm{mL}^{9}$ or a left ventricular ejection fraction $<50 \%$. Such patients were excluded to avoid the possible influence of these conditions on the transmitral inflow indexes. Clinical characteristics and echocardiographic parameters were evaluated while the patients were in normal sinus rhythm. Then they were classified into the group with progression to PeAF (PeAF group) or the group without progression (PAF group). Then we compared the groups to evaluate the factors that predicted progression from PAF to PeAF. And also, we divided all participants into three groups according to A-wave velocity. We evaluated the incident rate of the progression to PeAF in each group (figure 1).

\section{Echocardiography}

All patients underwent transthoracic M-mode, two-dimensional and Doppler echocardiography using a Toshiba ultrasound system. The left ventricular and left atrial dimensions were measured in M-mode according to the recommendations of the American Society of Echocardiography. ${ }^{10}$ After placing the cursor at the tips of the mitral valve leaflets in the apical four-chamber view, pulse-wave Doppler echocardiography was performed to obtain transmitral inflow images for measurement of the peak $\mathrm{E}$ and $\mathrm{A}$ wave velocities, as well as the ratio of these velocities (E/A). The deceleration time (DcT; the time between peak $\mathrm{E}$ and the intersection of the upper deceleration slope and the zero baseline) was also measured.

\section{Definitions}

PAF was defined as documentation of AF and subsequent resumption of sinus rhythm on the ECG following diagnosis by the referring physician, while PeAF was defined as documentation of AF on three consecutive ECGs obtained at intervals of at least 1 week. Hyperlipidaemia was defined by documentation of the diagnosis in the patient's medical record, current use of lipid-lowering agents, a fasting total cholesterol level $\geq 200 \mathrm{mg} /$ $\mathrm{dL}$ or a low-density lipoprotein cholesterol level $\geq 140 \mathrm{mg} / \mathrm{dL}$. Hypertension was defined as documentation of the diagnosis and/or use of antihypertensive medications. Diabetes mellitus was defined by the documentation of the diagnosis or treatment with oral hypoglycaemic agents or insulin.
Table 1 Clinical characteristics

\begin{tabular}{|c|c|c|c|}
\hline & $\operatorname{PeAF}(n=21)$ & $\operatorname{PAF}(n=67)$ & $P$ value \\
\hline Age (yrs) & $73.6 \pm 6.7$ & $73.7 \pm 6.7$ & 0.945 \\
\hline Sex (male; n, \%) & $14(66.7 \%)$ & $28(41.8 \%)$ & 0.0464 \\
\hline Body height $(\mathrm{cm})$ & $161.9 \pm 8.6$ & $158.7 \pm 8.2$ & 0.130 \\
\hline Body weight (kg) & $64.8 \pm 14.8$ & $60.0 \pm 13.7$ & 0.1786 \\
\hline BMI $\left(\mathrm{kg} / \mathrm{m}^{2}\right)$ & $24.6 \pm 4.7$ & $23.6 \pm 4.1$ & 0.3771 \\
\hline PQ time (ms) & $187.9 \pm 44.0$ & $179.6 \pm 32.8$ & 0.3569 \\
\hline E wave $(\mathrm{cm} / \mathrm{s})$ & $65.1 \pm 18.7$ & $66.1 \pm 17.6$ & 0.8235 \\
\hline A wave $(\mathrm{cm} / \mathrm{s})$ & $70.6 \pm 13.5$ & $80.7 \pm 18.2$ & 0.0224 \\
\hline$E / A$ & $0.988 \pm 0.46$ & $0.88 \pm 0.55$ & 0.4643 \\
\hline Dct (ms) & $228.2 \pm 43.1$ & $237.9 \pm 70.9$ & 0.5566 \\
\hline $\mathrm{e}^{\prime}(\mathrm{cm} / \mathrm{s})$ & $5.71 \pm 1.68$ & $6.32 \pm 1.61$ & 0.137 \\
\hline $\mathrm{E} / \mathrm{e}^{\prime}$ & $11.92 \pm 3.82$ & $10.80 \pm 2.96$ & 0.1621 \\
\hline $\operatorname{LVEF}(\%)$ & $65.6 \pm 4.8$ & $66.6 \pm 5.4$ & 0.4882 \\
\hline LAD (mm) & $40.5 \pm 5.0$ & $37.8 \pm 5.7$ & 0.0524 \\
\hline BNP (pg/mL) & $55.7 \pm 33.4$ & $53.4 \pm 43.4$ & 0.8217 \\
\hline WBC $(/ \mu \mathrm{L})$ & $6289.0 \pm 1250.3$ & $5891.2 \pm 1838.8$ & 0.3576 \\
\hline $\mathrm{Hb}(\mathrm{g} / \mathrm{dL})$ & $13.4 \pm 1.8$ & $13.5 \pm 1.4$ & 0.914 \\
\hline $\mathrm{Cr}(\mathrm{mg} / \mathrm{dL})$ & $0.83 \pm 0.23$ & $0.86 \pm 0.23$ & 0.6945 \\
\hline HTN & $15(71.4 \%)$ & $44(65.7 \%)$ & 0.6243 \\
\hline $\mathrm{HL}$ & $10(47.6 \%)$ & $34(50.8 \%)$ & 0.8025 \\
\hline DM & $5(23.8 \%)$ & $16(23.9 \%)$ & 0.995 \\
\hline$\beta$-blocker & $4(19.1 \%)$ & $9(13.4 \%)$ & 0.5269 \\
\hline Antiarrhythmics & $11(52.4 \%)$ & $39(58.2 \%)$ & 0.638 \\
\hline $\mathrm{CCB}$ & $12(57.1 \%)$ & $31(46.3 \%)$ & 0.3844 \\
\hline ARB/ACE-I & $12(57.1 \%)$ & $34(50.8 \%)$ & 0.6086 \\
\hline Anticoagulants & $13(61.9 \%)$ & $31(46.3 \%)$ & 0.2111 \\
\hline Smoking & 11 (52.4\%) & $22(32.8 \%)$ & 0.1065 \\
\hline Ischaemic stroke & $3(14.3 \%)$ & $5(7.5 \%)$ & 0.3426 \\
\hline
\end{tabular}

A, atrial inflow velocity; $A C E-I, A C E$ inhibitor; $A R B$, angiotensin receptor blocker; $B M I$, body mass index; BNP, brain natriuretic peptide; $C \mathrm{CB}$, calcium channel blocker; $\mathrm{Cr}$, creatinine; $\mathrm{DM}$, diabetes mellitus; DCT, deceleration time; $\mathrm{E}$, early inflow velocity; HL, hyperlipidaemia; HTN, hypertension; $\mathrm{Hb}$, haemoglobin; LAD, left atrial diameter; LVEF, left ventricular ejection fraction; PeAF, permanent atrial fibrillation; WBC, white blood cell.

\section{Statistical analysis}

Statistical analysis was performed with SPSS software. The mean and SD were calculated for continuous variables, while categorical variables were presented as percentages. To compare baseline characteristics between patients with or without progression to PeAF, we used an independent t-test for continuous variables and $\chi^{2}$ test for categorical variables. Analysis of covariance (ANCOVA) adjusted for confounding factors was performed to compare the measurements between groups. Receiver operating characteristic (ROC) curves were generated to determine the area under the curve (AUC), sensitivity and specificity for the predictive variables.

\section{RESULTS}

Of 88 patients enrolled, 21 patients $(23.9 \%)$ demonstrated progression from PAF to PeAF. Their baseline clinical characteristics, cardiovascular risk factors, laboratory data and echocardiographic parameters were presented in table 1. Compared with patients without progression to PeAF, patients with progression were more likely to be male. Patients in the PeAF group had a lower peak A velocity than the PAF group $(70.6 \pm 13.5 \mathrm{~cm} / \mathrm{s}$ vs $80.7 \pm 18.2 \mathrm{~cm} / \mathrm{s} ; \mathrm{p}=0.0224)$. There were no other significant differences in participant characteristics. ANCOVA was applied to compare the difference between the PeAF group and the PAF group, controlling for age, sex and body mass index (BMI). The 
Table 2 Echocardiographic parameters after adjustment

\begin{tabular}{lccl}
\hline & PeAF $(n=21)$ & $\operatorname{PAF}(n=67)$ & P value \\
\hline E wave $^{*}(\mathrm{~cm} / \mathrm{s})$ & $66.8 \pm 3.91$ & $65.6 \pm 2.16$ & 0.787 \\
A wave* $^{(\mathrm{cm} / \mathrm{s})}$ & $71.2 \pm 3.52$ & $80.5 \pm 1.95$ & 0.025 \\
$\mathrm{E} / A *^{*}$ & $1.00 \pm 0.119$ & $0.87 \pm 0.066$ & 0.341 \\
$\mathrm{DcT}^{*}(\mathrm{~ms})$ & $226.4 \pm 14.4$ & $238.5 \pm 7.9$ & 0.467 \\
$\mathrm{e}^{\prime *}(\mathrm{~cm} / \mathrm{s})$ & $5.79 \pm 0.332$ & $6.30 \pm 0.183$ & 0.189 \\
$\mathrm{E}^{\prime *}{ }^{*}$ & $12.08 \pm 0.604$ & $10.75 \pm 0.334$ & 0.059 \\
LVEF $^{*}(\%)$ & $65.6 \pm 1.15$ & $66.6 \pm 0.64$ & 0.487 \\
LAD* $^{*}(\mathrm{~mm})$ & $39.8 \pm 1.01$ & $38.0 \pm 0.56$ & 0.124 \\
\hline
\end{tabular}

The value is shown with adjusted average $\pm \mathrm{SE}$.

${ }^{*}$ Adjusted for age, sex and body mass index.

$A$, atrial inflow velocity; $D C T$, deceleration time; $E$, early inflow velocity; $L A D$, left atrial

diameter; LVEF, left ventricular ejection fraction; PeAF, permanent atrial fibrillation.

A velocity in the PeAF group remained significantly lower than the PAF group (table 2). The relation remained significant after controlling for other variables of interest (age, sex, BMI, PQ time, E velocity, DcT, e' velocity, left ventricular ejection fraction, left atrial diameter, hypertension, diabetes mellitus, antiarrhythmic drug, other medication and smoking). All participants were divided into three groups according to A-wave velocity: A1 ( $\mathrm{n}=29$, low velocity; $19.6-68.8 \mathrm{~cm} / \mathrm{s})$, A2 $(\mathrm{n}=29$, middle velocity; $69.2-85.6 \mathrm{~cm} / \mathrm{s})$ and A3 $(\mathrm{n}=30$, high velocity; $86.0-$ $128.5 \mathrm{~cm} / \mathrm{s}$ ). The incident rate of the progression to PeAF in the three categories of A-wave velocity is shown in figure 2. The incident rate in the group A3 was obviously lower than other groups. There were few differences in the incident rate in group $\mathrm{A} 1$ and group A2. The ROC analysis for the A velocity showed an AUC of $0.679(p=0.0032)$. The ROC curve revealed a cut-off A velocity value of approximately $75.3 \mathrm{~cm} / \mathrm{s}$ demonstrated sensitivity and specificity of $66.7 \%$ and $67.2 \%$, respectively.

\section{DISCUSSION}

In the present study, we performed an evaluation of predictive factors in the progression from PAF to PeAF including echocardiographic parameters. The progression rate of $23.9 \%$ was similar to the rate noted in previous studies. ${ }^{511}$ The present study demonstrated that the peak A velocity was independently associated with progression from PAF to PeAF in the people without underlying heart disease. Results suggested that the patients with

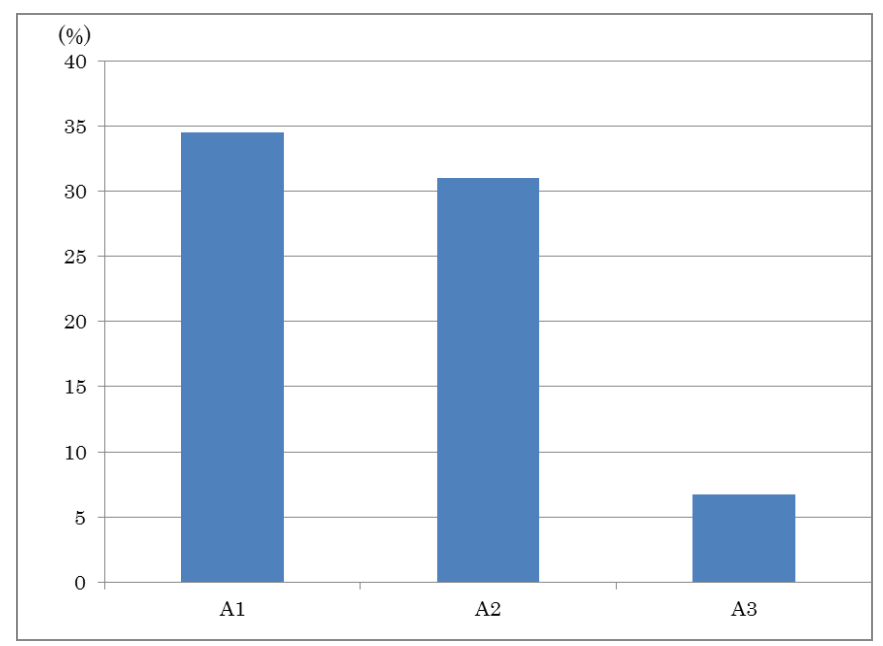

Figure 2 The incident rate of the progression to permanent atrial fibrillation in the three categories of $A$-wave velocity: $A 1$ : low $A$-wave velocity; $A 2$ : middle $A$-wave velocity; $A 3$ : high $A$-wave velocity.
PAF with the low A wave velocity were likely to progress to PeAF. The peak A velocity, a parameter routinely determined by echocardiography, could be used to predict the risk of progression to PeAF. Since transthoracic echocardiography is simple and non-invasive test, it is the ideal test for initial assessment of AF.

The A wave reflects atrial contraction and a decreased A wave velocity is associated with reduced atrial contraction. ${ }^{12}$ It has been reported that cardioversion of AF to sinus rhythm results in systolic stunning of the left atrium, and such left atrial stunning can markedly reduce the mitral A wave velocity in patients with recent cardioversion to sinus rhythm. ${ }^{13}$ It is thought that AF progresses to become PeAF because of structural and electrical remodelling of the atrium and that a decline of atrial contractility with remodelling results in a decrease of the A wave velocity. Previous study reported that a high A velocity indicated high atrial pressure and there was a U-shaped relation between AF development and the A wave. ${ }^{14}$ Other previous studies reported that $\mathrm{AF}$ progression was associated with $\mathrm{E}$ velocity or $\mathrm{E} / \mathrm{e}^{\prime}$ and not with A wave. These results are thought to be due to the rising pressure in left ventricular or left atrial chamber. To our knowledge, this is the first study to focus on the progression of the PeAF only in the patients aged 60 and over with the so-called 'idiopathic' AF. In this study, we restricted the participants who were considered to have high left ventricular or atrial pressure caused by underlying heart disease other than AF. Thus, we could evaluate the association between AF progression and transmitral inflow in the situation without pressure overload in left atrium caused by underlying heart disease. Left atrial diameter is a well-known predictor of AF development or recurrence. ${ }^{11} 1516$ Okçün et al reported that left atrial diameter greater than 45 $\mathrm{mm}$ had been associated with higher AF recurrence rate. ${ }^{17}$ In our study, left atrial size had no significant association with the progression to PeAF. This finding is mainly because left atrial size in both PAF group and PeAF group are not so large in this study. Furthermore, previous studies have identified several predictors of progression from PAF to PeAF, including age, left atrial diameter, significant aortic stenosis or mitral regurgitation, underlying cardiomyopathy and the heart rate during PAF. ${ }^{5} 1819$ Combining the A wave velocity with previously reported factors like age or the left atrial diameter might increase the accuracy of predicting the progression from PAF to PeAF.

The prevalence of AF is increasing dramatically and is estimated to be more than double in the next several decades. ${ }^{20}$ Previous studies described that as PAF progressed to more persistent form, the risk of thromboembolism, mortality and comorbidities, such as heart failure and stroke, were increasing. ${ }^{21}{ }^{22}$ And the patients became more frequently symptomatic, and AF became more uncontrollable. To identify the predictive factor for progression from PAF to PeAF can be helpful for early detection of AF progression and also to reduce the arrhythmia burden in patients with AF. With regard to the treatment of AF, the superiority of rhythm control therapy or rate control therapy has not been demonstrated and both strategies achieve similar results. ${ }^{23}$ Thus, we could maintain sinus rhythm safely by rhythm control therapy in patients who do not have an increased risk of progression to PeAF, improving their quality of life and prognosis. On the other hand, we could decrease prescription of antiarrhythmic agents and give priority to rate control therapy for patients with an increased risk of progression to PeAF.

\section{Limitations}

This was a retrospective study performed at a single centre, so it is impossible to eliminate bias. In addition, the restriction of participants (patients without underlying heart disease) limited 
our sample size. However, an advantage of this study design is that the present results might be applicable to similar populations. Finally, the definition of PeAF in this study is arbitrary. Prospective multicentre studies would be needed to obtain stronger evidence to confirm our findings.

\section{CONCLUSION}

This retrospective study suggests that low peak A velocity could predict the progression from PAF to PeAF. The simple parameter of echocardiography might be useful for the AF progression. The findings in this study would play an important role in the clinical practice such as diagnosis, treatment and prognosis of AF.

Contributors TN designed the study and wrote the initial draft of the manuscript. $\mathrm{HH}$ and YM contributed to analysis and interpretation of data and assisted in the preparation of the manuscript. All other authors have contributed to interpretation of data, critically reviewed the manuscript, approved the final version of the manuscript and agreed to be accountable for all aspects of the work in ensuring that questions related to the accuracy or integrity of any part of the work are appropriately investigated and resolved.

Funding The authors have not declared a specific grant for this research from any funding agency in the public, commercial or not-for-profit sectors.

Competing interests None declared.

Patient consent for publication Not required.

Ethics approval The study protocol was approved by the institutional ethics committee.

Provenance and peer review Not commissioned; externally peer reviewed.

Data sharing statement No data are available.

\section{REFERENCES}

1. Chugh SS, Havmoeller R, Narayanan K, et al. Worldwide epidemiology of atrial fibrillation: a global burden of disease 2010 study. Circulation 2014;129:837-47.

2. Feinberg WM, Blackshear JL, Laupacis $A$, et al. Prevalence, age distribution, and gender of patients with atrial fibrillation. analysis and implications. Arch Intern Med 1995; 155:469-73.

3. Psaty BM, Manolio TA, Kuller LH, et al. Incidence of and risk factors for atrial fibrillation in older adults. Circulation 1997;96:2455-61.

4. Fuster $V$, Rydén LE, Cannom DS, et al. ACC/AHA/ESC 2006 guidelines for the management of patients with atrial fibrillation: a report of the American College of Cardiology/American Heart Association Task Force on practice guidelines and the European Society of cardiology Committee for practice guidelines (writing Committee to revise the 2001 guidelines for the management of patients with atrial fibrillation): developed in collaboration with the European heart rhythm association and the heart rhythm Society. Circulation 2006;114:e257-354.

5. Kerr CR, Humphries $\mathrm{KH}$, Talajic $\mathrm{M}$, et al. Progression to chronic atrial fibrillation after the initial diagnosis of paroxysmal atrial fibrillation: results from the Canadian Registry of atrial fibrillation. Am Heart J 2005;149:489-96.
6. Allessie M, Ausma J, Schotten U. Electrical, contractile and structural remodeling during atrial fibrillation. Cardiovasc Res 2002;54:230-46.

7. Rosenberg MA, Gottdiener JS, Heckbert SR, et al. Echocardiographic diastolic parameters and risk of atrial fibrillation: the Cardiovascular Health study. Eur Heart J 2012;33:904-12.

8. Tsang TSM, Gersh BJ, Appleton CP, et al. Left ventricular diastolic dysfunction as a predictor of the first diagnosed nonvalvular atrial fibrillation in 840 elderly men and women. J Am Coll Cardiol 2002;40:1636-44.

9. Knudsen CW, Omland T, Clopton P, et al. Impact of atrial fibrillation on the diagnostic performance of B-type natriuretic peptide concentration in dyspneic patients: an analysis from the breathing not properly multinational study. J Am Coll Cardiol 2005;46:838-44.

10. Nagueh SF, Smiseth $O A$, Appleton CP, et al. Recommendations for the Evaluation of Left Ventricular Diastolic Function by Echocardiography: An Update from the American Society of Echocardiography and the European Association of Cardiovascular Imaging J Am Soc Echocardiogr 2016;29:277-314.

11. Nieuwlaat R, Prins MH, Le Heuzey J-Y, et al. Prognosis, disease progression, and treatment of atrial fibrillation patients during 1 year: follow-up of the Euro Heart survey on atrial fibrillation. Eur Heart J 2008;29:1181-9.

12. Plehn JF, Southworth J, Cornwell GG. Brief report: atrial systolic failure in primary amyloidosis. N Engl J Med 1992;327:1570-3.

13. Louie EK, Liu D, Reynertson SI, et al. "Stunning" of the left atrium after spontaneous conversion of atrial fibrillation to sinus rhythm: demonstration by transesophageal Doppler techniques in a canine model. J Am Coll Cardiol 1998:32:2081-6.

14. Vasan RS, Larson MG, Levy D, et al. Doppler transmitral flow indexes and risk of atrial fibrillation (the Framingham Heart study). Am J Cardiol 2003;91:1079-83.

15. Parkash R, Green MS, Kerr CR, et al. The association of left atrial size and occurrence of atrial fibrillation: a prospective cohort study from the Canadian Registry of atrial fibrillation. Am Heart J 2004;148:649-54.

16. DE Sisti A, Leclercq JF, Halimi $F$, et al. Evaluation of time course and predicting factors of progression of paroxysmal or persistent atrial fibrillation to permanent atrial fibrillation. Pacing Clin Electrophysiol 2014;37:345-55.

17. Okçün B, Yigit Z, Küçükoglu MS, et al. Predictors for maintenance of sinus rhythm after cardioversion in patients with nonvalvular atrial fibrillation. Echocardiography 2002;19:351-7.

18. Rienstra M, McManus DD, Benjamin EJ. Novel risk factors for atrial fibrillation: useful for risk prediction and clinical decision making? Circulation 2012;125:e941-6

19. Al-Khatib SM, Wilkinson WE, Sanders LL, et al. Observations on the transition from intermittent to permanent atrial fibrillation. Am Heart J 2000;140:142-5.

20. Go AS, Hylek EM, Phillips KA, et al. Prevalence of diagnosed atrial fibrillation in adults: national implications for rhythm management and stroke prevention: the anticoagulation and risk factors in atrial fibrillation (atria) study. JAMA 2001;285:2370-5

21. Ganesan AN, Chew DP, Hartshorne T, et al. The impact of atrial fibrillation type on the risk of thromboembolism, mortality, and bleeding: a systematic review and metaanalysis. Eur Heart J 2016;37:1591-602.

22. Chiang C-E, Naditch-Brûlé L, Murin J, et al. Distribution and risk profile of paroxysmal, persistent, and permanent atrial fibrillation in routine clinical practice: insight from the real-life global survey evaluating patients with atrial fibrillation international registry. Circ Arrhythm Electrophysiol 2012;5:632-9.

23. Wyse DG, Waldo AL, DiMarco JP, et al. A comparison of rate control and rhythm control in patients with atrial fibrillation. N Engl J Med 2002;347:1825-33. 